\title{
A pilot study of the in vitro antimicrobial activity and in vivo residual activity of chlorhexidine and acetic acid/boric acid impregnated cleansing wipes
}

\author{
Rebecca Rafferty ${ }^{1}$, Victoria H. Robinson ${ }^{2^{*}}$, Jennifer Harris ${ }^{1}$, Sally A. Argyle ${ }^{1}$ and Tim J. Nuttall ${ }^{1}$
}

\begin{abstract}
Background: Topical antimicrobials are recommended for first line treatment of surface and superficial infections in dogs. This is especially important given the increasing prevalence of antimicrobial resistant infections. Antimicrobial wipes have become popular, but there are a lack of controlled studies assessing their in vitro antimicrobial and in vivo residual activity. We aimed to assess the antimicrobial efficacy of two commercial antimicrobial wipes against frequently isolated pathogens.

Ten clinical and one reference isolate each of meticillin-susceptible Staphylococcus pseudintermedius (MSSP), meticillin-resistant S. pseudintermedius (MRSP), Escherichia coli (EC), extended spectrum beta-lactamase (ESBL) producing E. coli (ESBL-EC), Pseudomonas aeruginosa (PA) and Malassezia pachydermatis (MP) were tested using a modified Kirby-Bauer technique. Each isolate was tested against $6 \mathrm{~mm}$ discs of chlorhexidine (CHX) and acetic acid/boric acid (AABA) wipes, and positive and negative controls either overnight (bacteria) or for 3 days (Malassezia).

Healthy dogs were treated with the wipes and distilled water on a randomised flank ( $n=5$ each). Hair samples $(1 \mathrm{~cm} ; 0.1 \mathrm{~g})$ taken at days 0,1 and 3 were inoculated with an isolate of each organism. Zones of inhibition (ZI) were measured.

Results: All isolates produced confluent growth with AABA and control wipes, except for the cleansing wipes and MP (median ZI 12 mm; 95\% Cl 8.2-15.8). The median (95\% Cl) CHX wipe Zls (mm) were: MP 48.0 (47.0-49.0), MSSP 15.6 (14.2-17.0), MRSP 14.0 (13.6-14.4), EC 13.6 (12.0-15.2) and ESBL-EC 10.0 (9.4-10.6). PA showed confluent growth. The differences between the bacterial isolates was significant (Kruskal-Wallis $p<$ 0.0001; post-tests MSSP $=$ MRSP $=E C>E B S L-E C>P A)$. Confluent growth was visible with all the hair samples.

Conclusion: CHX but not AABA showed in vitro efficacy against MSSP, MRSP, EC and MP. ESBL-EC were less susceptible and there was no activity against PA. There was no residual activity on hair. Additional studies are required to determine efficacy of these products in clinically affected patients.
\end{abstract}

Keywords: Topical, Antimicrobial wipe, Chlorhexidine, Acetic acid, Boric acid, Microbial infection, Cutaneous, Dog

\footnotetext{
* Correspondence: tori.robinson@btconnect.com

${ }^{2}$ The Dermatology Referral Service, 528 Paisley Road West, Glasgow G51 1RN, UK

Full list of author information is available at the end of the article
}

(c) The Author(s). 2019 Open Access This article is distributed under the terms of the Creative Commons Attribution 4.0 International License (http://creativecommons.org/licenses/by/4.0/), which permits unrestricted use, distribution, and reproduction in any medium, provided you give appropriate credit to the original author(s) and the source, provide a link to the Creative Commons license, and indicate if changes were made. The Creative Commons Public Domain Dedication waiver (http://creativecommons.org/publicdomain/zero/1.0/) applies to the data made available in this article, unless otherwise stated. 


\section{Background}

Antimicrobial resistance (AMR) is a growing concern in human and veterinary healthcare. Treatment with systemic antibiotics, particularly broad-spectrum agents, is an important driver for the selection and dissemination of AMR. There is therefore renewed interest in using effective antiseptics to reduce systemic antibiotic use. For example, topical antiseptic therapy, particularly with chlorhexidine shampoos, is recommended as first line therapy for surface and superficial pyodermas in dogs $[1,2]$.

Antiseptic wipes are popular for their ease of use compared to shampoos [3], but it is important to know that the products are effective. Chlorhexidine ( $\mathrm{CHX})$ containing wipes $\left(\mathrm{CLX}^{\oplus}\right.$ wipes containing chlorhexidine, climbazole and trisEDTA; ICF, Cremona, Italy) and acetic acid/ boric acid (AABA) wipes (Malacetic ${ }^{\circledR}$ wipes containing acetic and boric acid; Dechra ${ }^{\oplus}$ Veterinary Products, Shrewsbury, UK) are both marketed as antiseptic, antibacterial and/or antifungal cleansing wipes. One study evaluated the in vivo and in vitro activity of $\mathrm{CHX}$ wipes against Malassezia pachydermatis in an experimental model with 5 shar pei dogs [4]. Applying the wipes 1 or 2 times daily to the skin for $30 \mathrm{~s}$ significantly reduced Malassezia pachydermatis counts on contact plates. In vitro assays using the wipe solution demonstrated complete kill in 6 isolates after 15 min contact time. However, antibacterial efficacy was not assessed and there were no control groups in the study. To our knowledge, there are no other peer-reviewed publication on the efficacy of these antiseptic wipes.

The aim of this pilot study was to assess the in vitro antimicrobial efficacy and residual in vivo antimicrobial activity of these wipes against meticillin-susceptible Staphylococcus pseudintermedius (MSSP), meticillinresistant S. pseudintermedius (MRSP), Escherichia coli (EC), extended spectrum beta-lactamase (ESBL) producing E. coli (ESBL-EC), Pseudomonas aeruginosa (PA) and Malassezia pachydermatis (MP) isolates. S. pseudintermedius and MP are commensals of canine skin and are frequently associated with microbial infections. PA, EC and ESBL-EC are less commonly isolated from cutaneous infections but they are important causes of ear, wound and surgical site infections [5-10].

\section{Results}

The positive control antimicrobial discs produced the expected ZIs (zones of inhibition) for susceptibility based on the breakpoints observed in their clinical identification and antimicrobial susceptibility testing. Colony morphology and cytology was consistent with pure cultures. There was no growth on any of the negative control plates (i.e. without an inoculum).

\section{Antimicrobial activity in vitro}

The CHX wipes produced significantly greater ZIs than the AABA wipes, paper towel or cleansing wipes for the MSSP, MRSP, EC, ESBL-EC and MP isolates (all $p<$ 0.0001 ; see Table 1) but there was no difference between the AABA wipes, paper towel and cleansing wipes where most isolates achieved confluent growth ( $p=0.05$ to 1.0$)$. All of the $P A$ isolates achieved confluent growth with the $\mathrm{CHX}$ and AABA wipes, paper towel and cleansing wipes. There was a significant difference between the ZIs for the CHX wipes among the different bacteria with $\mathrm{MSSP}=\mathrm{MRSP}=\mathrm{EC}>\mathrm{ESBL}-\mathrm{EC}>\mathrm{PA} \quad(p<0.0001) . \quad$ The ZIs for the reference isolates all fell within the ranges seen with the clinical isolates.

\section{Residual antimicrobial activity in vivo}

All the bacterial and Malassezia isolates achieved confluent growth around all the hair samples at days 0,1 and 3 .

\section{Discussion}

This study shows that the CHX wipes show in vitro efficacy against a range of pathogenic isolates relevant to skin and wound infections in animals, including MSSP, MRSP, EC, ESBL-EC and MP. The small differences between ZIs for MSSP, MRSP and EC were non-significant, but the

Table 1 Zones of inhibition (ZIs) of tested wipes. In vitro antimicrobial efficacy showing median (95\% Cl) of the zones of inhibition (Zls) (mm; Con = confluent growth) of the chlorhexidine (CHX) wipes, acetic acid/boric acid (AABA) wipes, paper towel and cleansing wipes (MSSP = meticillin-susceptible S. pseudintermedius; MRSP = meticillin-resistant S. pseudintermedius; EC =E. coli; ESBL$\mathrm{EC}=$ extended spectrum beta-lactamase producing $E$. coli; $\mathrm{PA}=$ Pseudomonas aeruginosa; $\mathrm{MP}=$ Malassezia pachydermatis; $n=11$ [10 clinical isolates and 1 Public Health England National Collection of Types Culture (NCTC) and Public Health England National Collection of Pathogenic Fungi (NCPF reference isolates])

\begin{tabular}{|c|c|c|c|c|c|c|}
\hline & MP & MSSP & MRSP & EC & ESBL-EC & $\mathrm{PA}$ \\
\hline CHX wipes & $\begin{array}{l}48.0 \\
(47.0-49.0\end{array}$ & $\begin{array}{l}15.6 \\
(14.2-17.0)\end{array}$ & $\begin{array}{l}14.0 \\
(13.6-14.4)\end{array}$ & $\begin{array}{l}13.6 \\
(12.0-15.2)\end{array}$ & $\begin{array}{l}10.0 \\
(9.4-10.6)\end{array}$ & Con \\
\hline AABA wipes & Con & Con & Con & Con & Con & Con \\
\hline Cleansing wipes & $\begin{array}{l}12 \\
(8.2-15.8)\end{array}$ & Con & Con & Con & Con & Con \\
\hline Paper towel & Con & Con & Con & Con & Con & Con \\
\hline
\end{tabular}


ESBL-EC showed significantly smaller ZIs and all the PA isolates achieved confluent growth. In contrast, all of the isolates achieved confluent growth when incubated with the AABA impregnated wipes. Neither wipe demonstrated any persistent activity on hair samples after thorough application in five dogs.

Chlorhexidine is an effective broad-spectrum antimicrobial [11-14]. Chlorhexidine containing shampoos, ear cleaners and sprays have shown in vivo and in vitro efficacy against a range of organisms including Pseudomonas [15-21]. The reason for the poor efficacy against PA (and to some extent ESBL-EC) in this study is unknown. Resistance to chlorhexidine has been associated with antibiotic resistance, which may be relevant to the multi-drug resistance seen in the PA and ESBL-EC isolates used in this study [22]. Kandry and colleagues showed that 22 out 36 MDR PA isolates carried class I integrons with reduced susceptibility to biocides including chlorhexidine. Furthermore, quaternary ammonium compound resistance E (qacE) genes, which are associated with chlorhexidine resistance, were identified in 11 isolates [22]. Nevertheless, while treatment failures are seen, these have not been proven to be associated with resistance to chlorhexidine in staphylococci and other bacteria in veterinary medicine. The relationship between resistance genes to clinical failure or success is therefore unclear.

Uri and others (2016) reported that the minimal inhibitory concentration (MIC) of MDR (multiple drug resistant) PA was $0.94 \mathrm{~g} / \mathrm{L}$ compared to the recommended concentration of $0.5 \mathrm{~g} / \mathrm{L}$. [23] The wipes in our study contain $0.3 \%(3 \mathrm{~g} / \mathrm{L})$ chlorhexidine, which is lower than that in many topical products (typically $2-4 \% ; 20-40 \mathrm{~g} /$ L). Nevertheless, in vitro inhibition has been demonstrated with a $0.15 \%$ chlorhexidine ear cleaner, and chlorhexidine has MIC and minimal bactericidal concentrations (MBCs) of $0.6-10 \mathrm{mg} / \mathrm{L}$ against the ESBL-EC and $5-10 \mathrm{mg} / \mathrm{L}$ against the PA isolates used in this study (data submitted for publication). MBCs of chlorhexidine to PA in previous veterinary studies have been found to be between 5 and $28 \mathrm{mg} / \mathrm{ml}[24,25]$ and MIC in human studies of $4 \mathrm{mg} / \mathrm{ml}$ [26]. which compares favourably to our submitted study. MICs for chlorhexidine against ESBL-EC in human clinical isolates was determined as $<1-2 \mathrm{mg} / \mathrm{L}$ $[26,27]$ and in avian isolates as $0.5-1 \mathrm{mg} / \mathrm{L}$. [27] MBCs of ESBL-EC were $7.32 \mathrm{mg} / \mathrm{L}$ ( $3 \mathrm{~min}$ incubation) to $1.83 \mathrm{mg} / \mathrm{L}$ (10 min incubation) with a $4 \%$ chlorhexidine product, and $468.75 \mathrm{mg} / \mathrm{L}$ (3 and $10 \mathrm{~min}$ incubations) with a $3 \%$ chlorhexidine $/ 0.5 \%$ climbazole product [23]. These additional studies have determined MIC/MBC comparable to the our submitted study.

Nonetheless, it is difficult to extrapolate the effective in vitro and in vivo concentration that bacteria would be exposed to using chlorhexidine-impregnated wipes. It is unknown how diffusion of the chlorhexidine, acetic acid/ boric acid, and other ingredients into the surrounding $\mathrm{MH}$ and SD agar affected the concentration that the organisms were exposed to. However, studies of the antimicrobial efficacy of products with these ingredients in agar well diffusion $[28,29]$ and agar plated hair models $[30,31]$ suggest that they freely diffuse into the media.

The CHX wipes also contained trisEDTA, zinc gluconate, climbazole and a range of other ingredients (Table 2). Their antimicrobial efficacy and/or effect on the efficacy of the chlorhexidine is unknown. TrisEDTA exhibits very little antimicrobial efficacy by itself, although it can potentiate the activity of chlorhexidine with $21 \%$ of isolates [13] particularly at high concentrations (at least $25-50 \mathrm{~g} / \mathrm{L}$ of a 4.2:1 combination TrisEDTA:chlorhexidine; data submitted for publication). It is unknown whether climbazole or chlorhexidine show additive or synergistic activity against MP, where the ZIs around the CHX disc were greater than the control antifungal disc. Fractional inhibition indices studies could be performed in future to determine if there is an additive or synergistic interaction between these compounds. Miconazole exhibits additive [13, 14] and synergistic [14] activity with chlorhexidine against some MSSP and MRSP isolates but is not known if this occurs with climbazole.

Table 2 Composition of the antimicrobial and control wipes according to the manufacturer's data

\begin{tabular}{|c|c|}
\hline $\begin{array}{l}\text { Chlorhexidine }(\mathrm{CHX}) \\
\text { wipes }\left(\mathrm{CLX}{ }^{\circledR} \text { wipes, ICF}{ }^{\oplus}\right. \\
\text { Cremona, Italy) }\end{array}$ & $\begin{array}{l}\text { 0.3\% Chlorhexidine } \\
0.5 \% \text { Climbazole } \\
\text { 1\% Zinc gluconate } \\
\text { TrisEDTA } \\
\text { Glycerin } \\
\text { Non-ionic surfactant } \\
\text { Benzyl alcohol } \\
\text { Propylene glycol } \\
\text { Perfume } \\
\text { Demineralized water }\end{array}$ \\
\hline $\begin{array}{l}\text { Acetic acid/boric acid } \\
\text { (AABA) wipes (Malacetic }{ }^{\circledast} \\
\text { wipes (Dechra }{ }^{\circledR} \text { Veterinary } \\
\text { Products, Shrewsbury, UK) }\end{array}$ & $\begin{array}{l}2 \% \text { Acetic acid } \\
2 \% \text { Boric acid } \\
\text { Propylene glycol } \\
\text { Glycerin } \\
\text { Fragrance }\end{array}$ \\
\hline $\begin{array}{l}\text { Simple }^{\circledR} \text { Kind to Skin Facial } \\
\text { Cleansing Wipes (Unilever, } \\
\text { Leatherhead, UK) }\end{array}$ & $\begin{array}{l}\text { Aqua } \\
\text { Benzoic acid } \\
\text { Cetearyl isononanoate } \\
\text { Ceteareth-12 } \\
\text { Ceteareth-20 } \\
\text { Cetearyl alcohol } \\
\text { Citric acid } \\
\text { Dehydroacetic acid } \\
\text { Disodium EDTA } \\
\text { Glycerin } \\
\text { Glyceryl stearate } \\
\text { Panthenol } \\
\text { Pantolactone } \\
\text { Phenoxyethanol } \\
\text { Sodium citrate } \\
\text { Tocopheryl acetate }\end{array}$ \\
\hline
\end{tabular}


No antimicrobial activity was seen with the AABA wipes. Similar findings have been reported for $2 \%$ acetic acid $/ 2 \%$ boric acid ear cleaners and shampoos in broth dilution studies [20, 21].Several human studies have looked at the antibacterial activity of acetic acid. MICs for PA and EC varied from $1.25-3.1 \mathrm{mg} / \mathrm{ml}$ [32-34] and $3.1 \mathrm{mg} / \mathrm{ml}$ for ESBL-EC [34]. This suggests that the antimicrobial activity of acetic acid varies within and between studies. There are few studies assessing boric acid, but reported MICs are $7.6 \mathrm{mg} / \mathrm{ml}$ against single isolates of EC and PA [35] and $0.385-0.77 \mathrm{mg} / \mathrm{ml}$ for two PA isolates [36] thus showing variation. Therefore, although acetic acid and boric acid are antimicrobial, dilution and/or interaction with other ingredients in the wipes may reduce the antimicrobial efficacy.

Our study also shows that there was no residual in vitro activity from hairs treated with these antiseptic wipes against the tested isolates. Only one isolate of each organism was tested, but we selected the isolate with the largest ZI to the $\mathrm{CHX}$ wipes to best show antimicrobial activity. The same isolates were used for AABA wipes as the earlier confluent growth prevented selection of an isolate in the same way. It is possible that there may have been some short-term activity as the first samples were collected $24 \mathrm{~h}$ after application. This study could have been improved by collecting a hair sample at shorter time intervals such as 30 mins. The authors are unaware of any short term clinical studies using this product. However, studies with identical methodology have shown that canine hairs show in vitro antimicrobial activity for up to 10 days after application of chlorhexidine containing shampoos and rinses [30, 31]. The reasons for the lack of persistent activity of the CHX wipes is unclear, but may relate to the low concentration of chlorhexidine in the formulation and further studies are indicated.

This study only used 10 clinical and one reference isolate of each organism for the in vitro experiment. Unfortunately, a reference isolate for MRSP was not available from NCTC at the time of this study. Ideally, larger numbers of clinical isolates would have been studied. However, the clinical isolates were chosen at random across different time periods to maximize representative sampling and avoid selection bias. The lack of bias is also supported by the narrow range of the ZIs (most of the $95 \%$ CI differed only $10 \%$ from the median results). Furthermore, the results for the reference isolates sat within the ranges established for the clinical isolates. Wide variability in the results would have suggested that much larger numbers of isolates would have been required to accurately demonstrate the relative in vitro antimicrobial activity of these products.

The study design was chosen to reflect the activity of the wipes rather than their ingredients, which have been established and would be less clinically relevant to the products as used. The experimental model was used to provide data on comparative in vitro antimicrobial activity. However, it is important to note that the model does not represent an in vitro disc diffusion test of the wipes and the results must not be read as breakpoints implying clinical susceptibility or resistance.

While these results demonstrate the in vitro antimicrobial activity of the $\mathrm{CHX}$, further studies are required to demonstrate clinical efficacy. This cannot be assumed, as, for example, Boonyasiri and colleagues [37] showed that there was no benefit to using $2 \%$ chlorhexidine impregnated wash cloths versus non-antimicrobial soap cloths for cleaning patients in a human ICU ward. The median time to MDR bacterial colonisation was 5 days with no significant difference in hospitalisation time or incidence of hospital acquired infections (including ESBL-EC, Meticillin -resistant Staphylococcus aureus, MDR Klebsiella pneumoniae and Acinetobacter baumanii) [37]. Recent meta-analysis by Patel and colleagues have found that there is no beneficial effect of daily use of chlorhexidine containing products to prevent gram negative bacterial infections caused by EC, PA, Acinetobacter, Klebsiella and Enterobacter [38].

\section{Conclusion}

This study has shown that CHX wipes inhibited the in vitro growth of a range of pathogens relevant to skin and wound infections in animals. AABA wipes were ineffective in vitro. Growth of the MSSP, MRSP, EC and MP isolates were inhibited, but the ESBL $E$. coli isolates appeared to be less susceptible and all the PA isolates achieved confluent growth. Clinicians should therefore use clinical signs, cytology, and where necessary culture, to determine whether treatment of an infection with the CHX product is appropriate. Finally, there was no residual activity on the hair suggesting that these $\mathrm{CHX}$ wipes need to be used at least once a day on active infections. Further studies are, however, required to establish whether these results can be replicated with larger samples and to demonstrate in vivo clinical efficacy.

\section{Methods \\ Microbial isolates}

Ten isolates each of MRSP, MSSP, EC, ESBL-EC, PA and MP were obtained from cases of canine otitis, pyoderma and wound infections. These isolates were randomly selected from samples submitted to the University of Edinburgh Veterinary Pathology Unit microbiology laboratory for routine microbial culture and susceptibility testing. Random numbers were computer generated to select samples using the laboratory submission numbers. Samples with insufficient material, growth characteristics and/or data were disregarded and the random selection process 
repeated until the target number was obtained. The organisms were speciated and their antimicrobial susceptibility established using standard methods employed by this accredited laboratory using CLSI guidelines [39, 40]. Reference isolates were obtained from the Public Health England National Collection of Type Cultures (NCTC) and Public Health England National Collection of Pathogenic Fungi (NCPF). These were Staphylococcus pseudintermedius NCTC 7151, E. coli NCTC 12241, CTX-M-15 ESBL E. coli NCTC 13353, Pseudomonas aeruginosa NCTC 10662 and Malassezia pachydermatis NCPF 3667. S. aureus EMRSA15 NCTC 13142 was used in the absence of an MRSP reference isolate. All the isolates were stored in tryptone soya broth (bacteria) or Sabouraud dextrose broth (Malassezia) with $15 \%$ glycerine at $-80^{\circ} \mathrm{C}$ until required. Isolates were defrosted and cultured overnight on Columbia 5\% horse blood agar (bacteria) at $37^{\circ} \mathrm{C}$ or for three days on Sabouraud dextrose agar (Malassezia) at $37^{\circ} \mathrm{C}$ with $5 \% \mathrm{CO}_{2}$. Colonies were washed and diluted in sterile phosphate buffered saline (PBS) to a visually assessed 0.5 McFarland standard. All the media were obtained from Oxoid ${ }^{\mathrm{m}}$ (ThermoFisher Scientific ${ }^{\mathrm{nm}}$, Basingstoke, UK).

\section{Agar diffusion studies}

The microbial isolates were spread onto Mueller-Hinton (MH; bacteria) and Sabouraud dextrose (SD; Malassezia) agar plates in a standard manner to achieve confluent growth. $6 \mathrm{~mm}$ discs were cut using sterile instruments from the chlorhexidine ( $\mathrm{CHX})$ and acetic acid/boric acid (AABA) impregnated wipes, and negative controls (sterile autoclaved Simple ${ }^{\circ}$ Kind to Skin Facial Cleansing Wipes [Unilever, Leatherhead, UK] and paper towel). See Table 2 for further details of the composition of the wipes. One disc of each was added to each plate. Antimicrobial impregnated discs to which the isolate had previously demonstrated susceptibility were used as positive controls. The choice of antimicrobial was based on previous culture and susceptibility testing performed to accepted CLSI guidelines [39, 40]. These included: $30 \mu \mathrm{g}$ cephalexin, $30 \mu \mathrm{g}$ doxycycline, $10 \mu \mathrm{g}$ gentamicin, $30 \mu \mathrm{g}$ amoxicillin/clavulanic acid, $30 \mu \mathrm{g}$ ceftazidime, $30 \mu \mathrm{g}$ amikacin, $5 \mu \mathrm{g}$ enrofloxacin and $25 \mu \mathrm{g}$ fluconazole (Oxoid $^{\mathrm{met}}$ ThermoFisher Scientific ${ }^{\mathrm{mm}}$, Basingstoke, UK). All the plates were set up in duplicate. Negative control MH and SD agar plates with the test, positive and negative control discs but without microbial isolates, were also included. Each isolate was incubated overnight (bacteria) or for 3 days (Malassezia) at $37{ }^{\circ} \mathrm{C}$ and $5 \% \mathrm{CO}_{2}$. The plates were then examined for microbial growth and the zones of inhibition (ZIs) were measured to the edge of the microbial growth. Colony morphology and cytological examination were used to determine the purity of the microbial growth.

\section{In vivo residual activity study}

Ethical approval through the parent institute was granted for this part of the study and all owners gave informed written consent. To evaluate the in vivo duration of activity, an area of haired skin on the lateral thorax in healthy dogs was vigorously rubbed with the $\mathrm{CHX}(n=5)$ or $\operatorname{AABA}(\mathrm{n}=5)$ wipes until the hair and underlying skin was thoroughly soaked. The other side of each dog was similarly treated with sterile distilled water. Treatment allocation to CHX or AABA and to the right or left side was randomly assigned using a coin toss with the investigator blinded to the allocation. No further topical treatment or grooming was permitted during the study period and the dogs were kept dry. Hair samples were collected from the treated sites at day 0 (i.e. before treatment), and then on days 1 and 3 . The hair was cut and weighed so that each sample was the same size $(1 \mathrm{~cm}$ length) and weight $(0.1 \mathrm{~g})$. Plates with an MSSP, MRSP, EC, ESBL-EC and MP isolate shown to be most susceptible to the CHX wipes were prepared as above. The PA isolate was selected at random as the isolates had all achieved confluent growth in the first part of the study. Each plate was then inoculated with the hair samples and positive control antimicrobial disc, and incubated as above. The ZI around each hair sample was examined and measured across the midpoint of the sample. Colony morphology and cytological examination were used to determine the purity of the microbial growth.

\section{Data analysis}

The ZIs were recorded in $\mathrm{mm}$. The median and 95\% confidence intervals (CIs) were calculated for the 11 isolates of each organism. Kruskal-Wallis tests with posttest analyses was used to compare the ZIs between the test and control wipes for each microbial isolate (i.e. CHX versus AABA for MSSP, MRSP, EC, ESBL-EC, PA and MP) and then between the ZIs for each wipe among the bacterial isolates (i.e. MSSP versus MRSP, EC, ESBL$\mathrm{EC}$ and PA for CHX and AABA). The MP isolates were not included in the latter analysis as these used a different culture methodology.

\section{Abbreviations \\ AABA: Acetic acid/boric acid; AMR: Antimicrobial resistance; CHX: Chlorhexidine; EC: Escherichia coli; ESBL-EC: Extended spectrum beta- lactamase (ESBL) producing E. coli; MBC: Minimal bactericidal concentrations; MDR: Multiple drug resistance; MIC: Minimal inhibitory concentration; MP: Malassezia pachydermatis; MRSP: Meticillin-resistant Staphylococcus. pseudintermedius; MSSP: Meticillin-susceptible Staphylococcus pseudintermedius; NCPF: Public Health England National Collection of Pathogenic Fungi; NCTC: Public Health England National Collection of Types Culture; PA: Pseudomonas aeruginosa; qacE: Quaternary ammonium compound resistance $\mathrm{E}$; Zl: Zones of inhibition}

\section{Acknowledgements}

We would like to thank the owners of the dogs used in the study and the veterinarians who submitted clinical microbial samples. We would also like to thank Helen Cobb, the Dermatology service nurse. A poster on this study was presented at 8th Dermatology World Conference in Bordeaux in 2016 as 
Rafferty R, Robinson V, Harris J, Argyle S, Nuttall T. A pilot study of the in vitro antimicrobial activity and in vivo residual activity of chlorhexidine and acetic acid/boric acid impregnated cleansing wipes. Vet Dermatol, 2016; 27 [1]: 87.

\section{Authors' contributions}

TN conceived the study and collected the hair samples. RR, VR and JH carried out laboratory based work. SA helped design the project, assisted with laboratory study and reviewed results. TN was responsible for statistical analysis. VR and TN drafted the manuscript. All authors read and approved the final manuscript.

\section{Funding}

This study was self-funded. ICF ${ }^{\oplus}$ paid the page charges for this article.

\section{Availability of data and materials}

The datasets used and analysed during the current study are available from the corresponding authors on reasonable request.

\section{Ethics approval and consent to participate}

This study was approved by the Royal (Dick) School of Veterinary Studies Ethics committee. Informed written consent was granted from the owners of the dogs. Clinical isolates were selected from archived samples at the Royal (Dick) School of Veterinary Studies Microbiology service.

\section{Consent for publication}

Not applicable.

\section{Competing interests}

TJ Nuttall and VH Robinson have received unrelated support and/or funding from ICF ${ }^{\oplus}$ and Dechra ${ }^{\bullet}$ Veterinary Products but the companies were not involved in the funding or the design of the study, and the authors have no financial or other interests in their products.

The other authors report no competing interests.

\section{Author details}

${ }^{1}$ University of Edinburgh, Royal (Dick) School of Veterinary Studies, Easter Bush Campus, Roslin EH25 9RG, UK. ${ }^{2}$ The Dermatology Referral Service, 528 Paisley Road West, Glasgow G51 1RN, UK.

\section{Received: 5 May 2019 Accepted: 20 September 2019}

\section{Published online: 30 October 2019}

\section{References}

1. Beco L, Guaguere E, Lorente Mendez C, Noli C, Nuttall T, Vroom M. Suggested guidelines for using systemic antimicrobials in bacterial skin infections (2): antimicrobial choice, treatment regimens and compliance. Vet Rec. 2013;172:156-60.

2. Hillier A, Lloyd DH, Weese JS, Blondeau JM, Boothe D, Breitschwerdt E, Guardabassi L, Papich M, Rankin S, Turnidge JD, Sykes JE. Guidelines for the diagnosis and antimicrobial therapy of canine superficial bacterial folliculitis (antimicrobial guidelines working Group of the International Society for companion animal infectious diseases). Vet Dermatol. 2014;25:163-e43.

3. Rosenkrantz W. Practical applications of topical therapy for allergic, infectious, and Seborrheic disorders. Clin Tech Small Anim Pract. 2006;21:106-16.

4. Cavana P, Peano A, Petit J-Y, Tizzabi P, Perrot S, Bensignor E, Guillot J. A pilot study of the efficacy of wipes containing chlorhexidine $0.3 \%$, climbazole $0.5 \%$ and Tris-EDTA to reduce Malassezia pachydermatis populations on canine skin. Vet Dermatol. 2015;26:278-e261.

5. Devriese LA, Vancanneyt M, Baele M, Vaneechoutte M, De Graef $E$, Snauwaert C, Cleenwerck I, Dawyndt P, Swings J, Decostere A, Haesebrouck F. Staphylococcus pseudintermedius sp. nov., a coagulase-positive species from animals. Int J Syst Evol Microbiol. 2005;55:1569-73.

6. Fortin $M$, Higgins R. Mixed infection associated with a group $B$ Streptococcus in a dog. Can Vet J. 2001:42:730

7. Hillier A, Alcorn JR, Cole LK, Kowalski JJ. Pyoderma caused by Pseudomonas aeruginosa infection in dogs: 20 cases. Vet Dermatol. 2006;17:432-9.

8. Summers JF, Hendricks A, Brodbelt DC. Prescribing practices of primary-care veterinary practitioners in dogs diagnosed with bacterial pyoderma. BMC Vet Res. 2014;10:240-50.

9. Chen T, Hill PB. The biology of Malassezia organisms and their ability to induce immune responses and skin disease. Vet Dermatol. 2005;16:4-26.
10. Bond R, Ferguson EA, Curtis CF, Craig JM, Lloyd DH. Factors associated with elevated cutaneous Malassezia pachydermatis populations in dogs with pruritic skin disease. J Small Anim Pract. 1996;37:103-7.

11. Moore SL, Payne DN. Types of Antimicrobial Agents In: Fraise AP, A. LP,Maillard JY, eds. Russell, Hugo \& Ayliffe's Principles and Practice of Disinfection, Preservation \& Sterilization. 4th edition. Oxford, UK: Blackwell Publishing Ltd. 2004; 8-97.

12. McDonnell G, Russell AD. Antiseptics and disinfectants: activity, action, and resistance. Clin Microbiol Rev. 1999;12:147-79.

13. Clark SM, Loeffler A, Schmidt VM, Chang Y-M, Wilson A, Timofte D, Bond R. Interaction of chlorhexidine with trisEDTA or miconazole in vitro against canine meticillin-resistant and -susceptible Staphylococcus pseudintermedius isolates from two UK regions. Vet Dermatol. 2016;27:340-e384.

14. Clark SM, Loeffler A, Bond R. Susceptibility in vitro of canine methicillinresistant and-susceptible staphylococcal isolates to fusidic acid, chlorhexidine and miconazole: opportunities for topical therapy of canine superficial pyoderma. J Antimicrob Chemother. 2015;70:2048-52.

15. Loeffler A, Cobb MA, Bond R. Comparison of a chlorhexidine and a benzoyl peroxide shampoo as sole treatment in canine superficial pyoderma. Vet Rec. 2011:169:249-53.

16. Guardabassi L, Ghibaudo G, Damborg P. In vitro antimicrobial activity of a commercial ear antiseptic containing chlorhexidine and Tris-EDTA. Vet Dermatol. 2010;21:282-6.

17. Murayama N, Terada Y, Okuaki M, et al. Dose assessment of $2 \%$ chlorhexidine acetate for canine superficial pyoderma. Vet Dermatol. 2011;22:449-53.

18. Viaud S, Maynard L, Sanquer A. Comparison of two shampoos as sole treatment for canine bacterial overgrowth syndrome. Vet Rec. 2012;170:675.

19. Borio S, Colombo S, La Rosa G, De Lucia M, Damborg P, Guardabassi L. Effectiveness of a combined (4\% chlorhexidine digluconate shampoo and solution) protocol in MRS and non-MRS canine superficial pyoderma: a randomized, blinded, antibiotic-controlled study. Vet Dermatol. 2015;26:339-e72.

20. Swinney A, Fazakerley J, McEwan N, Nuttall T. Comparative in vitro antimicrobial efficacy of commercial ear cleaners. Vet Dermatol. 2008;19:373-9.

21. Young R, Buckley L, McEwan N, Nuttall T. Comparative in vitro efficacy of antimicrobial shampoos: a pilot study. Vet Dermatol. 2012;23:36-e38.

22. Kadry AA, Serry FM, El-Ganiny AM, El-Baz AM. Integron occurrence is linked to reduced biocide susceptibility in multidrug resistant Pseudomonas aeruginosa. Br J Biomed Sci. 2017;74(2):78-84.

23. Uri M, Buckley LM, Marriage L, McEwan N, Schmidt VM. A pilot study comparing in vitro efficacy of topical preparations against veterinary pathogens. Vet Dermatol. 2016:27(3):152-e39.

24. Banovic F, Bozic F, Lemo N. In vitro comparison of the effectiveness of polihexanide and chlorhexidine against canine isolates of Staphylococcus pseudintermedius, Pseudomonas aeruginosa and Malassezia pachydermatis. Vet Dermatol. 2013;24:409-e89.

25. Ghibaudo G, Santospirito D, Sala A, Flisi S, Taddei S, Cavirani S, Cabassi CS. In vitro antimicrobial activity of a gel containing antimicrobial peptide AMP2041, chlorehexidine digluconate and Tris-EDTA on clinical isolates of Pseudomonas aeruginosa from canine otitis. Vet Dermatol. 2016;27:391-e98.

26. Grare M, Dibama HM, Lafosse S, Ribon A, Mourer M, Regnouf-de-Vains JB, Finance C, Duval RE. Cationic compounds with activity against multidrug-resistant bacteria: interest of a new compound compared with two older antiseptics, hexamidine and chlorhexidine. Clin Microbiol Infect. 2010;16(5):432-8.

27. Deus D, Krischek C, Pfeifer $Y$, Sharifi AR, Fiegen U, Reich F, Klein G, Kehrenberg C. Comparative analysis of the susceptibility to biocide and heavy metals of extended-spectrum- $\beta$ - lactamase-producing Escherichia coli isolates of human and avian origin. Germany Diagn Microbiol Infect Dis. 2017:88(1):88-92.

28. Mason CL, Steen SI, Paterson S, Cripps PJ. Study to assess in vitro antimicrobial activity of nine ear cleaners against 50 Malassezia pachydermatis isolates. Vet Dermatol. 2013;24:362-e81.

29. Steen SI, Paterson S. The susceptibility of Pseudomonas spp. isolated from dogs with otitis to topical ear cleaners. J Small Anim Prac. 2012;53:599-603.

30. Mesman ML, Kirby AL, Rosenkrantz WS, Griffen CE. Residual antibacterial activity of canine hair treated with topical antimicrobial sprays against Staphylococcus pseudintermedius in vitro. Vet Dermatol. 2016;27:261-e261.

31. Kloos I, Straubinger RK, Werckenthin C, Mueller RS. Residual antibacterial activity of dog hairs after therapy with antimicrobial shampoos. Vet Dermatol. 2013:24:250-4.

32. Agrawal K, Sarda A, Shrotriya R, Bachhav M, Puri V, Nataraj G. Acetic acid dressings: finding the holy grail for infected wound management. India J Plast Surg. 2017;50(3):273-80. 
33. Fraise AP, Wilkinson MAC, Bradley CR, Oppenheim B, Moiemen N. The antibacterial activity and stability of acetic acid. J Hosp Infect. 2013;84:329-31.

34. Halstead FD, Rauf M, Moiemen NS, Bamford A, Wearn CM, Fraise AP, Lund PA, Oppenheim BA, Webber MA. The antibacterial activity of acetic acid against biofilm-producing pathogens of relevance to burns patients. PLoS One. 2015;10:e0136190.

35. Yilmaz MT. Minimum inhibitory and minimum bactericidal concentrations of boron compounds against several bacterial strains. Turk J Med Sci. 2012; 42(2):1423-9.

36. Sayin Z, Ucan US, Sakmanoglu A. Antibacterial and antibiofilm effects of boron on different bacteria. Biol Trace Elem Res. 2016;173:241-6.

37. Boonyasiri A, Thaisiam P, Permpikul C, Judaeng $T$, Suiwongsa B, Apiradeewajeset N, Fakthongphan T, Suddee S, Laogtipparos W, Thamlikitkul V. Effectiveness of chlorhexidine wipes for the prevention of multidrug-resistant bacterial colonization and hospital-acquired infections in intensive care unit patients: a randomised trial in Thailand. Infect Control Hosp Epidermiol. 2016;37(3):245-53.

38. Patel A, Parikh P, Dunn AN, Otter JA, Thota P, Fraser TG, Donskey CJ, Deshpande A. Effectiveness of daily chlorhexidine bathing for reducing gram-negative infections: a meta-analysis. Infect Control Hosp Epidemiol. 2019;40(4):392-9.

39. Clinical and Laboratory Standards Institute. Performance standards for antimicrobial disk and dilution susceptibility tests for bacteria isolated from animals - approved standard $3^{\text {rd }}$ edition. CLSI document VET02-A3. 2008.

40. Clinical and Laboratory Standards Institute. Method for antifungal disk diffusion susceptibility testing of yeasts. 2nd edition. CLSI document M44. 2009.

\section{Publisher's Note}

Springer Nature remains neutral with regard to jurisdictional claims in published maps and institutional affiliations.

Ready to submit your research? Choose BMC and benefit from:

- fast, convenient online submission

- thorough peer review by experienced researchers in your field

- rapid publication on acceptance

- support for research data, including large and complex data types

- gold Open Access which fosters wider collaboration and increased citations

- maximum visibility for your research: over $100 \mathrm{M}$ website views per year

At $\mathrm{BMC}$, research is always in progress.

Learn more biomedcentral.com/submissions 